\title{
Scanning probe microscopy investigation of the bacteriophage effect on bacterial biofilms
}

Evgeny Dubrovin ${ }^{1,2}$, Natalia Kuzmina ${ }^{1}$, Ekaterina Varlamova ${ }^{1}$, Vasilii Kolmogorov ${ }^{2}$, Petr Gorelkin ${ }^{2}$, Alexander Erofeev ${ }^{2}$, Anastasia Popova ${ }^{3}$, Oleg Batishchev ${ }^{1}$

${ }^{1}$ A.N. Frumkin Institute of Physical Chemistry and Electrochemistry, Russian Academy of Sciences, Moscow, Russia, ${ }^{2}$ National University of Science and Technology MISIS, Moscow, Russia, ${ }^{3}$ State Research Center for Applied Microbiology and Biotechnology, Obolensk, Russia

\section{Background}

Biofilms are surface-associated bacterial communities immersed in a hydrated matrix of extracellular polymeric substance (EPS), comprised of polysaccharides, proteins, nucleic acids, and lipids (HallStoodley et al., 2004). Biofilms could grow on natural, industrial and hospital surfaces (Bridier et al., 2011), and are associated with up to $60 \%$ of all human infections (Spoering \& Lewis, 2001). At the same time polysaccharide capsule of bacterial biofilms increases their resistance to antibiotics (Stewart, 1996). Due to their ability to digest the EPS capsule of the host bacterial cell and reduce biofilms (Chai et al., 2014), depolymerase containing bacteriophages are considered as promising biofilm disruptive agents (Yan et al., 2014). Though many aspects of depolymerase associated bacteriophage induced biofilm degradation have been investigated, the physical mechanisms of this process remain not fully understood. The purpose of this work was direct investigation of bacteriophage effects on biofilms at a nano- and microscale using high-resolution scanning probe microscopy methods such as atomic force microscopy (AFM) and scanning ion conductance microscopy (SICM). The medically relevant A.baumannii cells and their specific bacteriophages were used in this study.

\section{Experimental}

Bacteriophage vB_AbaP_APK2 (APK2) (A.V. Popova et al., 2020) and its bacterial host, A. baumannii strain belonging to the capsular type K2 (Senchenkova et al., 2014) were used in this study. Biofilms were prepared by deposition of 50-1000 $\mu \mathrm{l}$ A. baumannii cells suspension in stationary or exponential growth phase on a substrate surface (bare or modified glass, mica, highly oriented pyrolytic graphite, polycarbonate) for 10 minutes -72 hours at $37^{\circ} \mathrm{C}$ or $21^{\circ} \mathrm{C}$. Then the samples were rinsed with distilled water followed by drying (for imaging in air) or addition of the imaging buffer (for imaging in liquid).

For AFM experiments we have used a multimode atomic force microscope with a Nanoscope V controller (Veeco, USA) operating in a tapping-mode using commercial cantilevers. AFM images were processed in Femtoscan software (Advanced Technologies Center, Russia). Biofilm topography measurements were performed in a Petri dish $(35 \mathrm{~mm})$ on scanning ion-conductive microscope (ICAPPIC Ltd, UK). Borosilicate nanocapillaries made of borosilicate blanks (Sutter Instruments, USA) with an outer diameter of $1.2 \mathrm{~mm}$, an inner diameter of $0.69 \mathrm{~mm}$ and a length of $7.5 \mathrm{~cm}$ were used as a probe.

\section{Results and Discussion}

Optical images of $A$. baumannii cells adsorbed on a glass surface demonstrate formation of biofilms already within 10 minutes after deposition (Figure 1b; the image is obtained in air). The surface area of biofilms ranges from 5 to $100 \mu \mathrm{m}^{2}$. Similar biofilms have been formed on mica, highly oriented pyrolytic graphite (HOPG) and polycarbonate surfaces. AFM images of A. baumannii biofilms obtained in air reveal tightly packed individual cells with round and slightly elongated shape (Figure 1b), which is typical for A. baumannii cells. The lateral size of individual cells varied from 0.5 to $1.2 \mu \mathrm{m}$ depending on the growth conditions, whereas the height was considerably lower, in the range of $200-700 \mathrm{~nm}$, depending on 
preparation, indicative of cell shrinkage caused by sample drying. Typical SICM images of A. baumannii biofilm obtained in Hanks solution (Figure 1c) demonstrate the biofilm height of $\sim 1 \mu \mathrm{m}$ and multiple values, which correspond to one and several bacterial layers, respectively.

Exposition of bacterial biofilms to bacteriophage containing solution leads to changes in the biofilm morphology and appearance of adsorbed bacteriophage particle on bacterial surface (Figure 2a). The apparent packaging of individual cells in a biofilm becomes sparser. Also under certain conditions biofilms become rich in small holes $\sim 40-100 \mathrm{~nm}$ in diameter. These observations may be associated with digestion of EPS capsule by bacteriophages. Incubation of $A$. baumannii biofilms with bacteriophages for 25 minutes at $37^{\circ} \mathrm{C}$ leads to a complete destruction of some cells (e.g., top left cells in Figure $2 b$ ) that is connected with a cell lysis caused by bacteriophage (the lytic cycle of this bacteriophages is 20 minutes). However, the major fraction of the cells remains undistorted due to small multiplicity of infection and peculiarities of lytic cycle in the adsorbed bacterial cells (Abraham et al., 2020).

\section{Conclusions}

A. baumannii biofilms formed on different substrates have been characterized using AFM and SICM. Specific bacteriophages induce apparent changes of $A$. baumannii biofilms such as EPS capsule digestion, bacteriophage adsorption and cell lysis.

\section{Acknowledgements}

The reported study was funded by RFBR, project number 20-34-70040, and by the Ministry of Education and Science of the Russian Federation in the framework of increase Competitiveness Program of NUST "MISIS", implemented by a governmental decree dated 16th of March 2013, No 211.

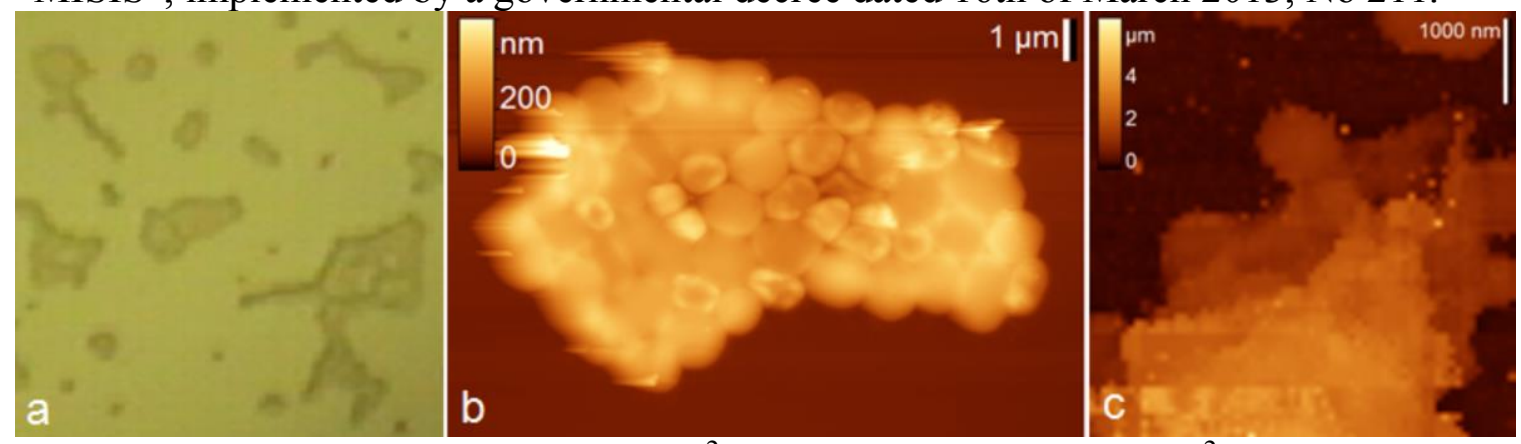

Figure 1. (a) Optical image $\left(100 \times 100 \mu \mathrm{m}^{2}\right)$, (b) AFM image $\left(15 \times 10 \mu \mathrm{m}^{2}\right)$, and (c) SICM image $\left(5 \times 5 \mu \mathrm{m}^{2}\right)$ of $A$. baumannii biofilm formed on a glass surface after $(\mathrm{a}, \mathrm{b}) 10$ minutes, (c) 24 hours of incubation with bacterial cell suspension in $\mathrm{M} 9$ medium at $21^{\circ} \mathrm{C}$.

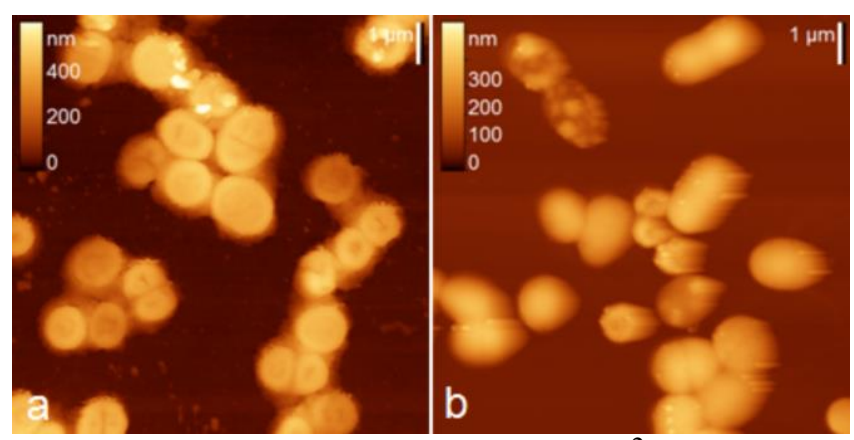

Figure 2. AFM images $\left(10 \times 10 \mu \mathrm{m}^{2}\right)$ of $A$. baumannii biofilm on a glass surface after exposition to bacteriophage solution for (a) 5 minutes (b) 25 minutes at $37^{\circ} \mathrm{C}$.

References 
Abraham, S., Kaufman, Y., Perreault, F., Young, R. \& Bar-Zeev, E. (2020). Bursting out: linking changes in nano-topography and biomechanical properties of biofilm-forming Escherichia coli to T4 lytic cycle. bioRxiv 2020.06.29.176883.

A.V. Popova, Shneider, M. M., Arbatsky, N. P., Kasimova, A. A., Senchenkova, S. N., Shashkov, A. S., Dmitrenok, A. S., Chizhov, A. O., Mikhailova, Y. V., Shagin, D. A., Sokolova, O. S., Timoshina, O. Y., Kozlov, R. S., Miroshnikov, K. A. \& Knirel, Y. A. (2020). Specific interaction of novel Friunavirus phages encoding tailspike depolymerases with corresponding Acinetobacter baumannii capsular types. Journal of Virology.

Bridier, A., Briandet, R., Thomas, V. \& Dubois-Brissonnet, F. (2011). Resistance of bacterial biofilms to disinfectants: a review. Biofouling 27, 1017-1032.

Chai, Z., Wang, J., Tao, S. \& Mou, H. (2014). Application of bacteriophage-borne enzyme combined with chlorine dioxide on controlling bacterial biofilm. LWT - Food Science and Technology 59, 1159-1165.

Hall-Stoodley, L., Costerton, J. W. \& Stoodley, P. (2004). Bacterial biofilms: from the Natural environment to infectious diseases. Nature Reviews Microbiology 2, 95-108.

Senchenkova, S. N., Shashkov, A. S., Shneider, M. M., Arbatsky, N. P., Popova, A. V., Miroshnikov, K. A., Volozhantsev, N. V. \& Knirel, Y. A. (2014). Structure of the capsular polysaccharide of Acinetobacter baumannii ACICU containing di-N-acetylpseudaminic acid. Carbohydrate Research 391, 89-92.

Spoering, A. L. \& Lewis, K. (2001). Biofilms and Planktonic Cells of Pseudomonas aeruginosa Have Similar Resistance to Killing by Antimicrobials. Journal of Bacteriology 183, 6746-6751.

Stewart, P. S. (1996). Theoretical aspects of antibiotic diffusion into microbial biofilms. Antimicrobial Agents and Chemotherapy 40, 2517-2522.

Yan, J., Mao, J. \& Xie, J. (2014). Bacteriophage Polysaccharide Depolymerases and Biomedical Applications. BioDrugs 28, 265-274. 